\title{
Expression of Selected Epithelial-Mesenchymal Transition Transcription Factors in Endometrial Cancer
}

\author{
Paweł Sadłecki (D), 1 Jakub Jóźwicki $\left(D,{ }^{2}\right.$ Paulina Antosik $\left(\mathbb{D},{ }^{2}\right.$ \\ and Malgorzata Walentowicz-Sadłecka $\left(^{1}{ }^{1}\right.$ \\ ${ }^{1}$ Department of Obstetrics and Gynecology, Collegium Medicum in Bydgoszcz, Nicolaus Copernicus University, Torun, \\ Bydgoszcz, Poland \\ ${ }^{2}$ Department of Clinical Pathomorphology, Collegium Medicum in Bydgoszcz, Nicolaus Copernicus University, Torun, \\ Bydgoszcz, Poland
}

Correspondence should be addressed to Paweł Sadłecki; pawelsadlecki@cm.umk.pl

Received 28 July 2020; Accepted 18 December 2020; Published 29 December 2020

Academic Editor: Plamen Todorov

Copyright (C) 2020 Paweł Sadłecki et al. This is an open access article distributed under the Creative Commons Attribution License, which permits unrestricted use, distribution, and reproduction in any medium, provided the original work is properly cited.

Endometrial cancer (EC) is the most common gynecologic malignancy in developed countries. The aim of this study was to analyze the expression of SNAIL, SLUG, TWIST1, TWIST2, ZEB1, and ZEB 2 in primary tumor and the correlation with morphological and clinical characteristics of EC. The study included 158 patients with EC after surgical treatments: total hysterectomy and lymphadenectomy. The percentages of EC specimens testing positively for the EMT transcription factors were 84.5\% for SNAIL, 92.2\% for SLUG, $10.9 \%$ for TWIST1, 100\% for TWIST2, 89\% for ZEB1, and 98\% for ZEB2. The expression of SLUG in patients with FIGO stage III or IV, type II EC, myometrial invasion $\geq 50 \%$ of the uterine wall thickness, and adnexal involvement and in patients with distant metastases was significantly higher. SLUG and ZEB2 expressions were identified as significant predictors of higher FIGO stages (III or IV) on univariate analysis. The overexpression of SLUG was a significant predictor of more aggressive type II EC, myometrial invasion $\geq 50 \%$ of the uterine wall thickness, and distant metastases on both univariate and multivariate analysis. Moreover, the overexpression of SLUG and ZEB2 was shown to be significant predictors of adnexal involvement on univariate analysis. ZEB 2 overexpression was identified in multivariate analysis as another independent predictor associated with a lesser likelihood of type II EC. Both univariate and multivariate analyses demonstrated that SLUG expression was the only predictor of 5-year survival in the study group. The overexpression of SLUG was associated with a significant increase in mortality hazard on univariate analysis and was shown to be a highly significant predictor of death on multivariate analysis. Conclusions. Selected proteins of the EMT pathway play a role in endometrial carcinogenesis; SLUG and ZEB2 expressions in the primary tumor might predict clinical outcomes in EC and drive therapeutic decisions regarding adjuvant treatment in patients with this malignancy.

\section{Introduction}

Endometrial cancer (EC) is the most common gynecologic malignancy in developed countries, representing nearly 5\% of all female cancers and being responsible for more than $2 \%$ of cancer-related deaths in women worldwide [1]. The incidence and mortality of EC have been increasing in recent years and are expected to increase even further [2-5]. In 75\% of patients, EC is diagnosed after menopause and in 15\% during their reproductive years $[6,7]$. Most ECs are detected at early stages when a curative resection is a feasible option in most cases. While 5-year survival rate in such patients approximates $95 \%$, it decreases down to $16-45 \%$ in women with advanced late-stage EC [8-10].

EC can be classified into two types, type I and type II. Type I EC is endometrioid cancer (G1 or G2) with a hormonal etiology. The more aggressive type (type II EC) is a G3 malignancy with endometrioid, clear cell, or serous phenotype [11]. The type II tumors are estrogen-independent and associated with endometrial atrophy [12]. Most of type 
I ECs have a significantly better prognosis than the type II malignancies $[13,14]$. Type I EC usually has a characteristic clinical profile; it is found in younger patients, most of whom have an identifiable source of excess estrogen, and endometrioid histologic subtypes are overwhelmingly more common [15]. The foremost risk factor for endometrial cancer, obesity, is strongly related to circulating estrogen levels in postmenopausal women and seems to be associated with an increased risk of EC of any type [16, 17]. An increase in the relative risk of EC in younger patients has been estimated at 1.59 per $5 \mathrm{~kg} / \mathrm{m}^{2}$ BMI [18]. Other hormone-related risk factors for EC include nulliparity and older age at menopause $[19,20]$. The list of presumable protective factors includes oral contraceptives, cigarette smoking, and older age at the last birth $[20,21]$. Compared with women with type I EC, patients with type II EC are older, which more often have normal body weight and non-Caucasian heritage [16].

The majority of EC patients are eligible for primary surgical treatment: total hysterectomy or salpingo-oophorectomy, combined with lymphadenectomy if warranted by the presence of relevant risk factors [22]. The examination of surgical specimens provides information about cancer stage, prognosis, and the necessity of adjuvant treatment to decrease the likelihood of recurrence in patients with highrisk tumors [23]. EC is often spread via the lymphatics, with lymphatic metastases found in approximately $25 \%$ of patients with G3 tumors. Myometrial invasion up to twothirds of the uterine wall thickness and vascular invasion are associated with the lymphatic spread in $22 \%$ and $15 \%$ of the cases, respectively. While only pelvic lymph nodes are involved in two-thirds of patients with lymphatic spread, more than $10 \%$ of women with EC may present with isolated positive para-aortal nodes [24]. Thus, hysterectomy with bilateral adnexectomy and pelvic and para-aortal lymphadenectomy is a recommended treatment option in patients with G3 tumors or/and myometrial invasion exceeding $50 \%$ of the uterine wall thickness [24-26]. In women with G1 or G2 tumors and more superficial myometrial invasion, hysterectomy with bilateral adnexectomy, but without lymphadenectomy, is a sufficient treatment option [27]. Radiotherapy is considered an effective adjuvant treatment option in EC [28].

Epithelial-mesenchymal transition (EMT) is a reversible cellular process during which epithelial cells depolarize, lose cell-cell contacts, and gain a spindle-mesenchymal morphology. EMT and an opposite process, mesenchymal to epithelial transition (MET), are essential for several developmental events in the embryo, such as gastrulation, formation of neural crest cells from the neural tube, mesoderm and heart valve formation, and palatogenesis [29]. EMT is associated with the loss of epithelial morphology and cytoskeletal reorganization, which allows the cells to gain migratory potential and higher invasiveness. Aside from being a physiological developmental mechanism, EMT also plays a role in tumor growth and progression to metastatic cancer. The EMT is triggered by several transcription factors in different signaling pathways. Several transcription factors, among them SNAIL, SLUG, TWIST, and ZEB, were shown to play essential roles in EMT control, contributing to downregulation of E-cadherin. The loss of Ecadherin expression is considered a major event in the EMT.
TABLE 1: Clinical characteristics of study subjects.

\begin{tabular}{|c|c|c|c|}
\hline Feature & & $N$ & $\%$ \\
\hline \multirow{4}{*}{ Stage } & I & 75 & 47 \\
\hline & II & 34 & 22 \\
\hline & III & 38 & 24 \\
\hline & IV & 11 & 7 \\
\hline \multirow{3}{*}{ Grade } & 1 & 9 & 6 \\
\hline & 2 & 105 & 72 \\
\hline & 3 & 32 & 22 \\
\hline \multirow{2}{*}{ Bokham subtype } & I & 118 & 75 \\
\hline & II & 40 & 25 \\
\hline \multirow{2}{*}{ LVSI } & Negative & 129 & 82 \\
\hline & Positive & 29 & 18 \\
\hline \multirow{2}{*}{ Meta at lymph nodes } & No & 124 & 82 \\
\hline & Yes & 28 & 18 \\
\hline \multirow{2}{*}{ Distant metastases } & No & 137 & 91 \\
\hline & Yes & 14 & 9 \\
\hline \multirow{2}{*}{ Infiltration of myometrium } & $<50 \%$ & 48 & 30 \\
\hline & $\geqq 50 \%$ & 110 & 70 \\
\hline \multirow{2}{*}{ Infiltration of cervix } & No & 95 & 61 \\
\hline & Yes & 62 & 39 \\
\hline \multirow{2}{*}{ Infiltration of adnexa } & No & 134 & 87 \\
\hline & Yes & 20 & 13 \\
\hline
\end{tabular}

LVSI: lymphovascular space invasion.

As compared with other malignancies, still little is known about the role of EMT transcription factors in EC [30]; the aim of this study was to analyze the expression of these factors: SNAIL, SLUG, TWIST1, TWIST2, ZEB1, and ZEB 2 in primary EC. Moreover, we verified whether the expression of the EMT transcription factors correlated with morphological and clinical characteristics of EC.

\section{Methods}

The study included 158 patients with EC, diagnosed, and treated at the Department of Obstetrics and Gynecology, Nicolaus Copernicus University, Collegium Medicum in Bydgoszcz (Poland). The mean age of the study patients was 66 years (range 37-87 years). The mean age at the last menstruation was 51 years (range 37-59 years), and the median parity amounted to 2 (range $0-10$ ). The mean BMI for the study group was $29.56 \mathrm{~kg} / \mathrm{m}^{2}$ (range $19-42 \mathrm{~kg} / \mathrm{m}^{2}$ ). Clinicopathological characteristics of the study patients are summarized in Table 1. All patients underwent surgical treatment: total hysterectomy and lymphadenectomy. Adjuvant treatment consisted of radiotherapy, administered in line with the guidelines of Polish Oncological Society [31]. The relationship between the protein expression and survival was analyzed after minimum 5-year follow-up. Immunohistochemical studies were performed on archival formalinfixed, paraffin-embedded (FFPE) tissue specimens, obtained 
from the Department of Clinical Pathomorphology Collegium Medicum, Nicolaus Copernicus University in Torun, Poland.

2.1. Tissue Macroarrays. Representative tumor areas were selected from the archival paraffin blocks (donor blocks). Tissue macroarrays (TMaAs) were obtained by transferring tissue fragments from the donor blocs to previously prepared recipient blocs. Each recipient block was composed of representative tissue from five EC patients. The representative tissue was reembedded in paraffin to obtain recipient blocks. Next, the TMaA block was cut into $4 \mu \mathrm{m}$ thick sections, using a rotary microtome (Accu-Cut ${ }^{\circledR}$ SRM $^{\mathrm{TM}} 200$; Sakura, Torrance, CA, USA). The sections were transferred on extraadhesive slides (Superfrost Plus; Mensel-Glazer, Braunschweig, Germany) and left for one hour on a heating plate set at $60^{\circ} \mathrm{C}$.

2.2. Immunohistochemical Staining (IHC). Immunohistochemical staining was performed according to the protocol described previously [32], using the primary rabbit polyclonal anti-SNAIL ( $1: 100,45 \mathrm{~min}, \mathrm{NBP} 1-80022$, Novus Biologicals) antibody, mouse monoclonal anti-SLUG (1:200, 30 min, NBP2-03886, Novus Biologicals) antibody, mouse monoclonal anti-TWIST1 (1:20, $60 \mathrm{~min}, \mathrm{ab50887}$, Abcam) antibody, mouse monoclonal anti-TWIST2 (1:200, $45 \mathrm{~min}$, ab57997, Abcam) antibody, rabbit polyclonal anti-ZEB1 (1:500, $30 \mathrm{~min}$, HPA027524, Sigma-Aldrich) antibody, and rabbit polyclonal anti-ZEB2 (1:100, $45 \mathrm{~min}$, HPA003456, Sigma-Aldrich) antibody. The antibody complexes were detected using an EnVision Flex Anti-Mouse/Rabbit HRPLabeled Polymer (Dako; Agilent Technologies, Inc.) and localized using 3,3'-diaminobenzidine (DAB) as the chromogen. The staining for SNAIL, SLUG, TWIST1, TWIST2, ZEB1, and ZEB2 was performed automatically in AutostainerLink48 (Dako; Agilent Technologies, Inc.). To standardize the immunohistochemical procedures, a series of positive and negative control reactions were carried out. Positive controls were tissue models in which the presence of analyzed antigens was indicated in reference sources (The Human Protein Atlas: https://www.proteinatlas.org.) as well as in the respective antibody datasheet. Negative control was obtained by substituting the primary antibody with a $1 \%$ bovine serum albumin (BSA) diluted in phosphatebuffered saline (PBS).

2.3. Evaluation of Immunohistochemical Reactions. The antibody-labeled slides were evaluated by two independent pathologists under a low-power $(\times 20)$ ECLIPSE E800 light microscope (Nikon Instruments Europe, Amsterdam, Netherlands). In addition, low and negative-intensity slides were evaluated in high-power magnification (x40). The immunoexpression of analyzed proteins in EC macroarrays was quantified using Remmele-Stegner (IRS) scoring system. The IRS score for each macroarray spot was calculated by multiplying staining intensity $(0=$ negative, $1=$ weakly positive, 2 =moderately positive, and $3=$ strongly positive) by the proportion of positively stained cells $(1=1-9 \%, 2=$ $10-50 \%, 3=51-80 \%$, and $4=81-100 \%)$; hence, the final scores might vary between 0 and 12. Statistical analysis included a mean IRS score for all tissue macroarrays [33].

2.4. Statistical Analysis. Statistical analysis was carried out with PQStat package, version 1.6.4.110. The effect of grouping clinicopathological variables on the immunoexpression of studied proteins was analyzed with the Mann-Whitney $U$-test. The relationship between analyzed proteins and established unfavorable prognostic factors in EC was estimated using univariate and multivariate logistic regression models. The relationship between the protein expression and survival was analyzed with univariate and multivariate Cox proportional-hazards models. Survivals of patients with weaker and stronger expressions of SLUG were compared with log-rank, Wilcoxon-Breslow-Gehan, and Taron-Ware tests. The results of all tests were considered significant at $p$ $<0.05$ and highly significant at $p<0.01$.

2.5. Ethics. The protocol of the study was approved by the Local Bioethics Committee at the Nicolaus Copernicus University, Collegium Medicum in Bydgoszcz.

\section{Results}

In this study, the immunoexpressions of several EMT proteins: SLUG, SNAIL, TWIST1, TWIST2, ZEB1, and ZEB2, were evaluated in primary ECs. The percentages of EC specimens testing positively for the EMT transcription factors were $84.5 \%$ for SNAIL, $92.2 \%$ for SLUG, $10.9 \%$ for TWIST1, $100 \%$ for TWIST2, $89 \%$ for ZEB1, and $98 \%$ for ZEB2. In the case of SNAIL, SLUG, TWIST1, and ZEB1, the immunoexpression was found in the cell nuclei and in the case of TWIST2 and ZEB2 in the cytoplasm. Representative immunohistochemical reactions for various proteins are shown in Figures 1-6.

The expression of SLUG differed significantly $(p<0.05)$ depending on clinical FIGO stage. The expression in patients with FIGO stage III or IV was significantly higher than that in those with less-advanced ECs. The SLUG expression was also significantly higher $(p<0.01)$ in type II ECs than in type I malignancies. No statistically significant differences in the SLUG expression were found after stratifying the immunohistochemical results according to histological grade, lymphovascular space invasion (LVSI), cervical invasion, and lymph node involvement. The expressions of SLUG in patients with myometrial invasion $\geq 50 \%$ of the uterine wall thickness and adnexal involvement were significantly higher $(p<0.05)$ than those in those without these unfavorable prognostic factors. The expression of SLUG was also significantly higher in patients with distant metastases $(p<0.05)$ (Table 2). No significant associations were found between the clinicopathological characteristics of EC patients and the expressions of TWIST1, TWIST2, ZEB1, and SNAIL.

ZEB2 expression was significantly higher in patients with adnexal involvement than in those without. Other clinicopathological variables (FIGO stage, Bokhman type, histological grade, LVSI, myometrial invasion $\geq 50 \%$ of the uterine wall thickness, cervical invasion, lymph node involvement, 


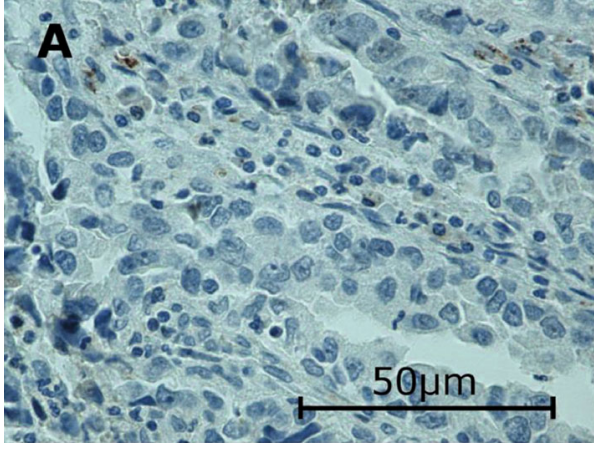

(a)

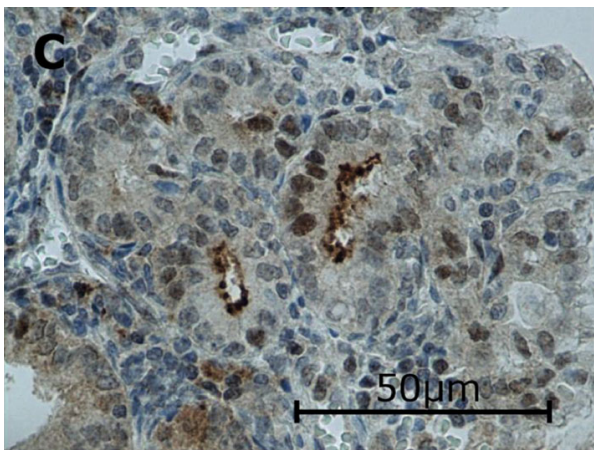

(c)

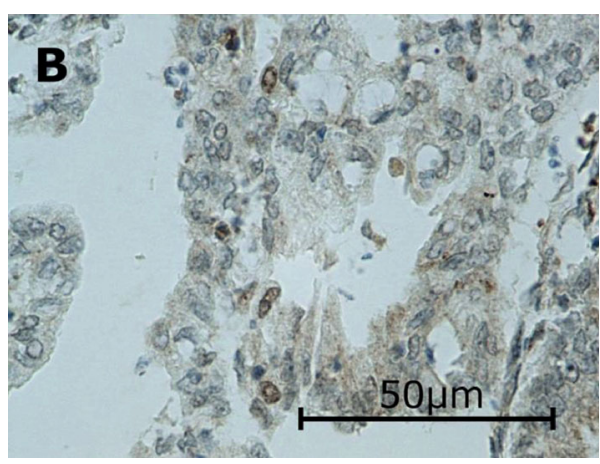

(b)

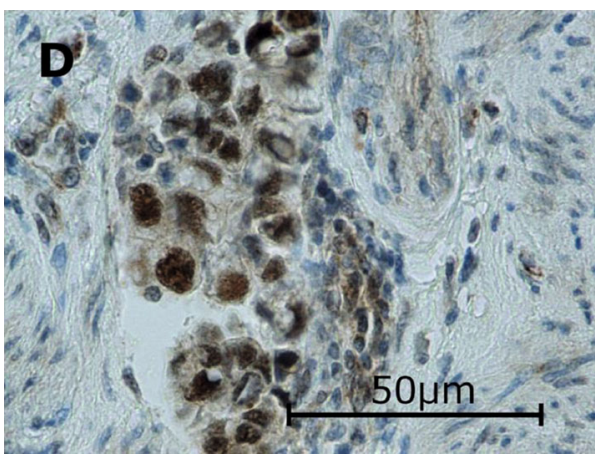

(d)

FIGURE 1: Levels of nuclear SNAIL expression in endometrial cancer: negative (a), weak (b), medium (c), and strong (d). Primary objective magnification: $40 \mathrm{x}$.

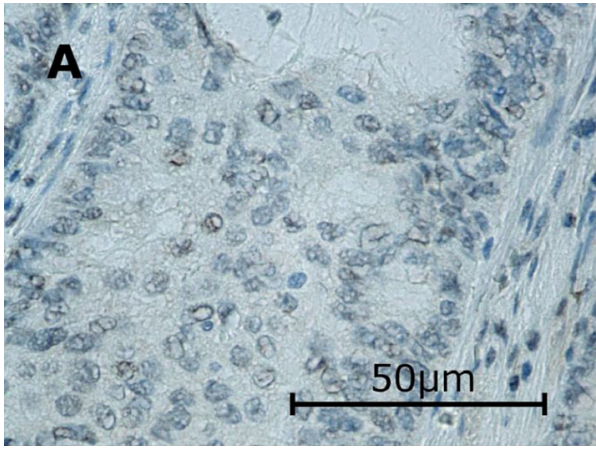

(a)

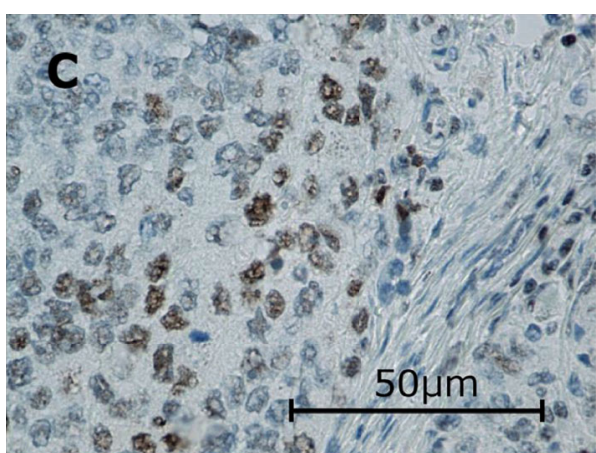

(c)

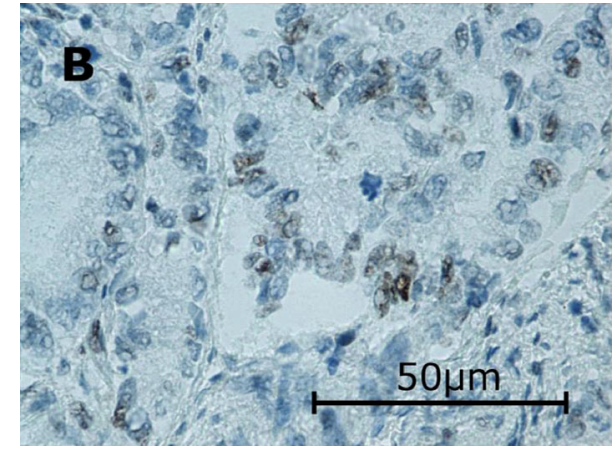

(b)

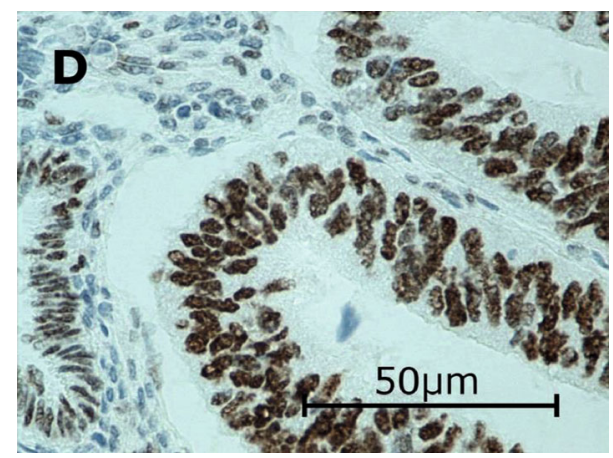

(d)

FIGURE 2: Levels of nuclear SLUG expression in endometrial cancer: negative (a), weak (b), medium (c), and strong (d). Primary objective magnification: 40x. 


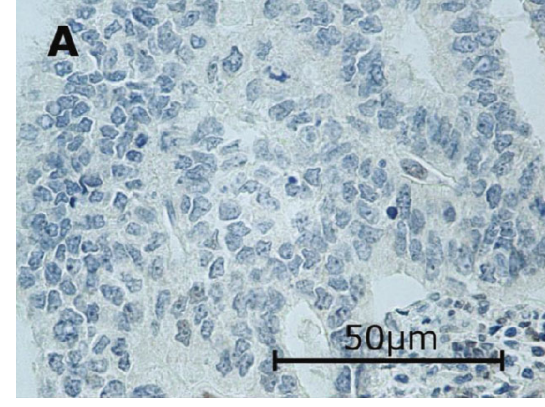

(a)

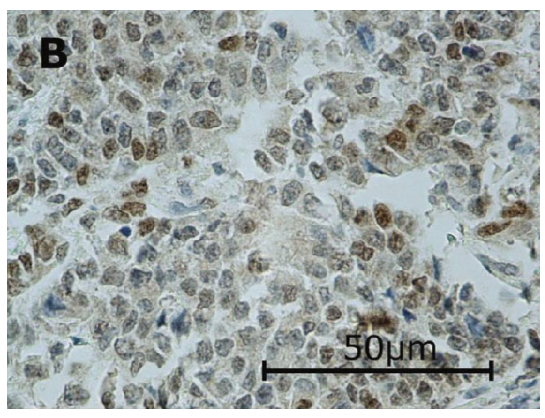

(b)

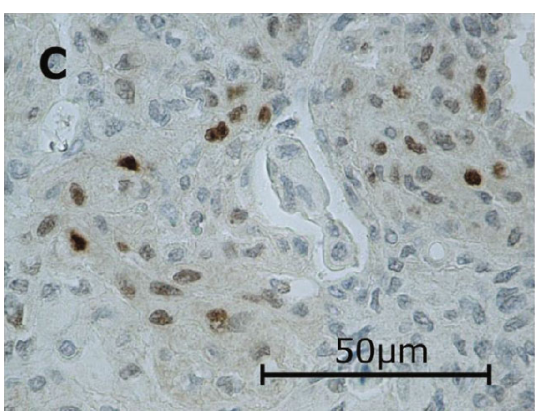

(c)

FIgURE 3: Levels of nuclear TWIST1 expression in endometrial cancer: negative (a), medium (b), and strong (c). Notice that although the conspicuous expression, it is always in few cells. There was no weak expression in our study. Primary objective magnification: 40x.

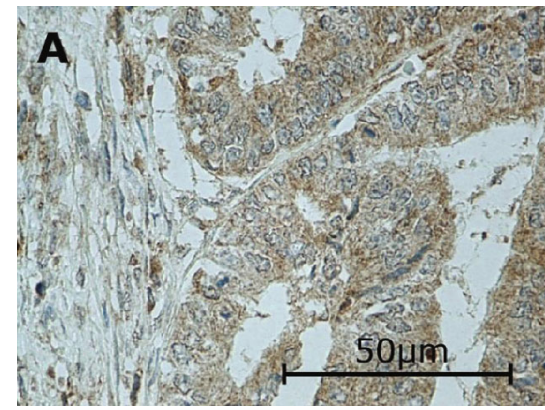

(a)

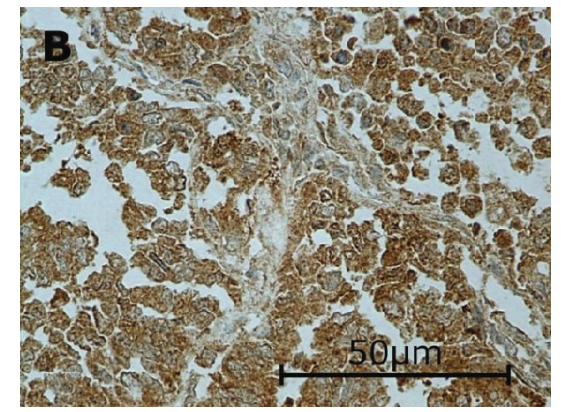

(b)

FIGURE 4: Levels of cytoplasmic TWIST2 expression in endometrial cancer: weak (a) and medium (b). There was neither negative nor strong expression in our study. Primary objective magnification: 40x.

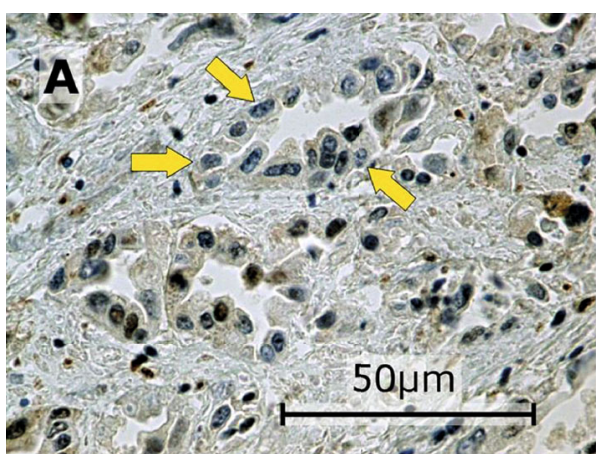

(a)

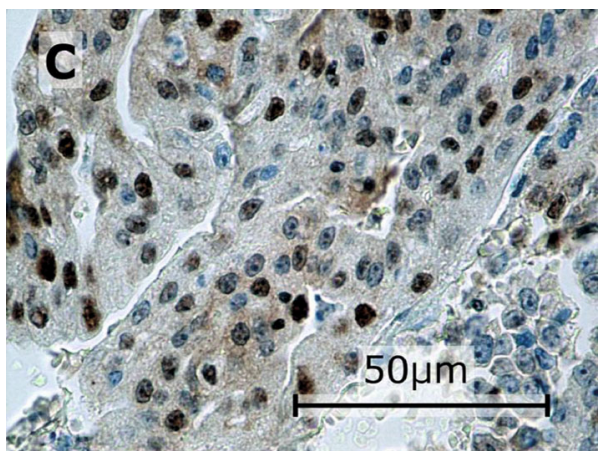

(c)

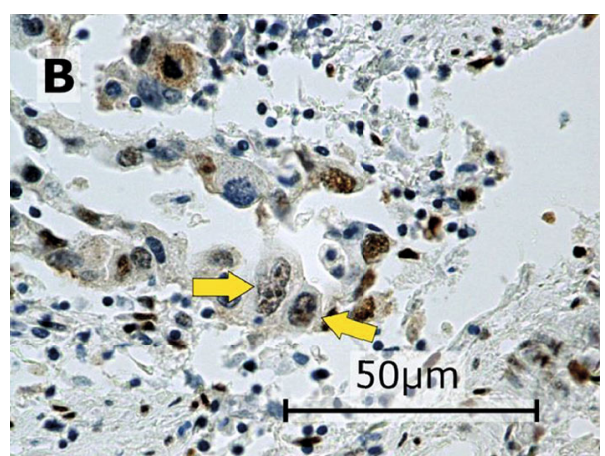

(b)

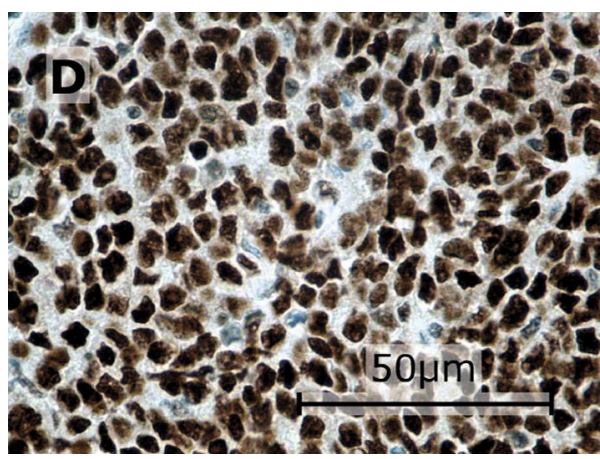

(d)

FIgURE 5: Levels of nuclear ZEB1 expression in endometrial cancer: negative ((a) arrows), weak ((b) arrows), medium (c), and strong (d). Primary objective magnification: 40x. 


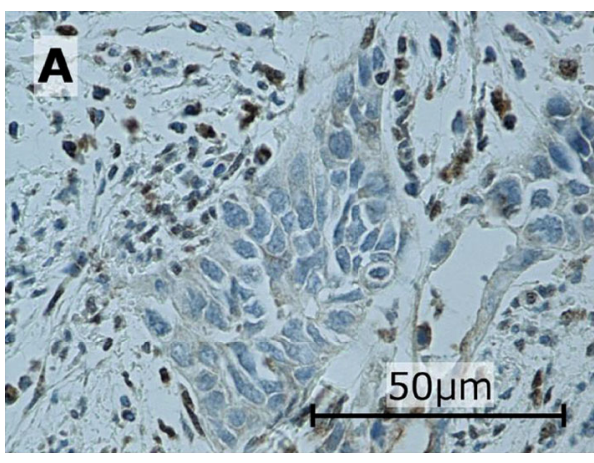

(a)

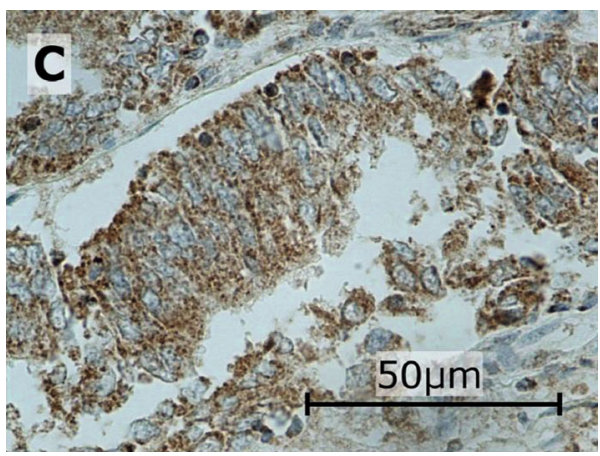

(c)

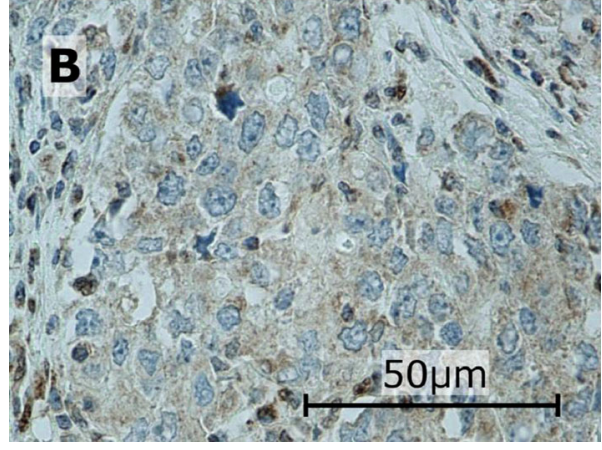

(b)

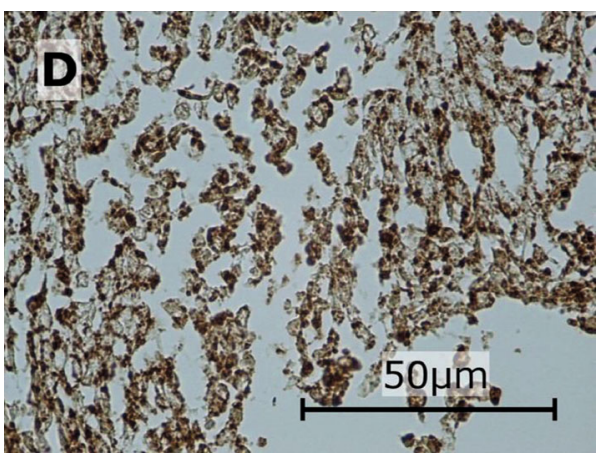

(d)

FIgURE 6: Levels of cytoplasmic ZEB2 expression in endometrial cancer: negative (a), weak (b), medium (c), and strong (d). Primary objective magnification: 40x.

TABLE 2: SLUG expression according to clinicopathological features.

\begin{tabular}{|c|c|c|c|c|c|c|c|c|c|}
\hline & & Mean & $\begin{array}{c}\text { Standard } \\
\text { deviation }(\mathrm{SD})\end{array}$ & Minimum & $\begin{array}{c}\text { Lower } \\
\text { quartile (Q1) }\end{array}$ & $\begin{array}{c}\text { Median } \\
(\mathrm{Me})\end{array}$ & $\begin{array}{c}\text { Upper } \\
\text { quartile (Q3) }\end{array}$ & Maximum & $\begin{array}{c}\text { Mann- } \\
\text { Whitney } U \text {-test }\end{array}$ \\
\hline Total & & 7.30 & 3.93 & 0 & 4 & 8 & 12 & 12 & - \\
\hline \multirow{2}{*}{ FIGO stage } & $\mathrm{I}+\mathrm{II}$ & 6.82 & 3.85 & 0 & 4 & 7 & 9 & 12 & \multirow{2}{*}{$\begin{array}{l}Z=2.3333 \\
p=0.0196\end{array}$} \\
\hline & $\mathrm{III}+\mathrm{IV}$ & 8.35 & 3.94 & 0 & 6 & 8.5 & 12 & 12 & \\
\hline \multirow{2}{*}{$\begin{array}{l}\text { Bokhman } \\
\text { subtype }\end{array}$} & I & 6.64 & 3.85 & 0 & 4 & 6 & 9 & 12 & \multirow{2}{*}{$\begin{array}{l}Z=3.7265 \\
p=0.0002\end{array}$} \\
\hline & II & 9.18 & 3.57 & 0 & 8 & 10.5 & 12 & 12 & \\
\hline \multirow{2}{*}{ Grading } & $\mathrm{G} 1+\mathrm{G} 2$ & 7.08 & 3.98 & 0 & 4 & 8 & 12 & 12 & \multirow{2}{*}{$\begin{array}{l}Z=0.6527 \\
p=0.5140\end{array}$} \\
\hline & G3 & 7.68 & 3.97 & 0 & 4 & 8 & 12 & 12 & \\
\hline \multirow{2}{*}{ LVSI } & Positive & 7.15 & 3.94 & 0 & 4 & 8 & 12 & 12 & \multirow{2}{*}{$\begin{array}{l}Z=0.9659 \\
p=0.3341\end{array}$} \\
\hline & Negative & 7.96 & 3.91 & 0 & 6 & 8 & 12 & 12 & \\
\hline \multirow{2}{*}{$\begin{array}{l}\text { Myometrial } \\
\text { invasion }\end{array}$} & $<50 \%$ & 6.04 & 3.92 & 0 & 3.25 & 6 & 9 & 12 & \multirow{2}{*}{$\begin{array}{l}Z=2.4943 \\
p=0.0126\end{array}$} \\
\hline & $\geq 50 \%$ & 7.83 & 3.83 & 0 & 4 & 8 & 12 & 12 & \\
\hline \multirow{2}{*}{$\begin{array}{l}\text { Cervical } \\
\text { involvement }\end{array}$} & No & 6.86 & 4.00 & 0 & 4 & 8 & 12 & 12 & \multirow{2}{*}{$\begin{array}{l}Z=1.6616 \\
p=0.0966\end{array}$} \\
\hline & Yes & 8.00 & 3.78 & 0 & 4 & 8 & 12 & 12 & \\
\hline \multirow{2}{*}{$\begin{array}{l}\text { Infiltration of } \\
\text { adnexa }\end{array}$} & No & 7.05 & 3.91 & 0 & 4 & 8 & 12 & 12 & \multirow{2}{*}{$\begin{array}{c}Z=2.135 \\
p=0.0328\end{array}$} \\
\hline & Yes & 9.00 & 3.39 & 0 & 7.5 & 9 & 12 & 12 & \\
\hline \multirow{2}{*}{$\begin{array}{l}\text { Lymph nodes } \\
\text { metastases }\end{array}$} & No & 6.94 & 3.81 & 0 & 4 & 8 & 9 & 12 & \multirow{2}{*}{$\begin{array}{c}Z=1.757 \\
p=0.0789\end{array}$} \\
\hline & Yes & 8.33 & 4.43 & 0 & 5 & 12 & 12 & 12 & \\
\hline \multirow{2}{*}{$\begin{array}{l}\text { Distant } \\
\text { metastases }\end{array}$} & No & 6.99 & 3.99 & 0 & 4 & 8 & 12 & 12 & \multirow{2}{*}{$\begin{array}{c}Z=2.5126 \\
p=0.012\end{array}$} \\
\hline & Yes & 9.86 & 2.66 & 6 & 8 & 12 & 12 & 12 & \\
\hline
\end{tabular}

FIGO: Federation Internationale de Gynecologie et d'Obstetrique; LVSI: lymphovascular space invasion. 
TABLE 3: ZEB 2 expression according to clinicopathological features.

\begin{tabular}{|c|c|c|c|c|c|c|c|c|c|}
\hline & & Mean & $\begin{array}{c}\text { Standard } \\
\text { deviation }(\mathrm{SD})\end{array}$ & Minimum & $\begin{array}{c}\text { Lower } \\
\text { quartile }(\mathrm{Q} 1)\end{array}$ & $\begin{array}{l}\text { Median } \\
\text { (me) }\end{array}$ & $\begin{array}{c}\text { Upper } \\
\text { quartile (Q3) }\end{array}$ & Maximum & $\begin{array}{c}\text { Mann-Whitney } \\
U \text {-test }\end{array}$ \\
\hline Total & & 5.13 & 2.02 & 0 & 4 & 4 & 8 & 8 & - \\
\hline \multirow{2}{*}{ FIGO stage } & $\mathrm{I}+\mathrm{II}$ & 4.91 & 2.02 & 0 & 4 & 4 & 7 & 8 & \multirow{2}{*}{$\begin{array}{l}Z=1.9126 \\
p=0.0558\end{array}$} \\
\hline & $\mathrm{III}+\mathrm{IV}$ & 5.63 & 1.95 & 3 & 4 & 4 & 8 & 8 & \\
\hline \multirow{2}{*}{$\begin{array}{l}\text { Bokhman } \\
\text { subtype }\end{array}$} & I & 5.04 & 1.97 & 0 & 4 & 4 & 8 & 8 & \multirow{2}{*}{$\begin{array}{l}Z=1.2604 \\
p=0.2075\end{array}$} \\
\hline & II & 5.65 & 2.11 & 2 & 4 & 4 & 8 & 8 & \\
\hline \multirow{2}{*}{ Grading } & $\mathrm{G} 1+\mathrm{G} 2$ & 5.25 & 2.09 & 0 & 4 & 4 & 8 & 8 & \multirow{2}{*}{$\begin{array}{l}Z=1.4442 \\
p=0.1487\end{array}$} \\
\hline & G3 & 4.78 & 1.78 & 1 & 4 & 4 & 4.5 & 8 & \\
\hline \multirow{2}{*}{ LVSI } & Positive & 5.20 & 2.09 & 0 & 4 & 4 & 8 & 8 & \multirow{2}{*}{$\begin{array}{l}Z=0.9575 \\
p=0.3383\end{array}$} \\
\hline & Negative & 4.79 & 1.66 & 2 & 4 & 4 & 4.5 & 8 & \\
\hline \multirow{2}{*}{$\begin{array}{l}\text { Myometrial } \\
\text { invasion }\end{array}$} & $<50 \%$ & 5.08 & 2.01 & 0 & 4 & 4 & 8 & 8 & \multirow{2}{*}{$\begin{array}{l}Z=0.5308 \\
p=0.5955\end{array}$} \\
\hline & $\geq 50 \%$ & 5.41 & 1.97 & 3 & 4 & 4 & 8 & 8 & \\
\hline \multirow{2}{*}{$\begin{array}{l}\text { Cervical } \\
\text { involvement }\end{array}$} & No & 5.11 & 2.00 & 0 & 4 & 4 & 8 & 8 & \multirow{2}{*}{$\begin{array}{l}Z=0.5294 \\
p=0.5965\end{array}$} \\
\hline & Yes & 5.50 & 2.03 & 3 & 4 & 4 & 8 & 8 & \\
\hline \multirow{2}{*}{$\begin{array}{l}\text { Infiltration of } \\
\text { adnexa }\end{array}$} & No & 5.22 & 2.01 & 1 & 4 & 4 & 8 & 8 & \multirow{2}{*}{$\begin{array}{l}Z=0.2918 \\
p=0.7704\end{array}$} \\
\hline & Yes & 5.09 & 2.03 & 0 & 4 & 4 & 8 & 8 & \\
\hline \multirow{2}{*}{$\begin{array}{l}\text { Lymph nodes } \\
\text { metastases }\end{array}$} & No & 4.92 & 1.90 & 0 & 4 & 4 & 6 & 8 & \multirow{2}{*}{$\begin{array}{l}Z=1.7265 \\
p=0.0843\end{array}$} \\
\hline & Yes & 5.46 & 2.17 & 0 & 4 & 4 & 8 & 8 & \\
\hline \multirow{2}{*}{$\begin{array}{l}\text { Distant } \\
\text { metastases }\end{array}$} & No & 5.01 & 2.01 & 0 & 4 & 4 & 8 & 8 & \multirow{2}{*}{$\begin{array}{l}Z=2.1077 \\
p=0.0351\end{array}$} \\
\hline & Yes & 6.00 & 1.95 & 4 & 4 & 6 & 8 & 8 & \\
\hline
\end{tabular}

FIGO: Federation Internationale de Gynecologie et d'Obstetrique; LVSI: lymphovascular space invasion.

TABLE 4: Results of univariate logistic regression analyses examining the effects of selected proteins on the incidence of advanced FIGO stage (FIGO III+IV).

\begin{tabular}{lccccc}
\hline & $b$ coefficient & $p$ value & Odds ratio & $-95 \%$ CI & $+95 \%$ CI \\
\hline Intercept & $-1,144$ & 0,0527 & 0,3186 & 0,1001 & 1,0135 \\
TWIST 2 & 0,0532 & 0,5414 & 1,0546 & 0,8891 & 1,251 \\
Intercept & $-0,7336$ & $<0,0001$ & 0,4802 & 0,3376 & 0,683 \\
TWIST 1 & $-0,2509$ & 0,2406 & 0,7781 & 0,5118 & 1,1831 \\
Intercept & $-1,588$ & 0,0001 & 0,2043 & 0,091 & 0,4588 \\
SLUG & 0,1046 & 0,0264 & 1,1103 & 1,0123 & 1,2177 \\
Intercept & $-1,7366$ & 0,0005 & 0,1761 & 0,0659 & 0,471 \\
ZEB 2 & 0,1777 & 0,0417 & 1,1945 & 1,0067 & 1,4173 \\
Intercept & $-0,5327$ & 0,138 & 0,587 & 0,2904 & 1,1868 \\
ZEB 1 & $-0,0623$ & 0,3993 & 0,9396 & 0,813 & 1,086 \\
Intercept & $-0,5927$ & 0,0709 & 0,5528 & 0,2906 & 1,0518 \\
SNAIL & $-0,0548$ & 0,4799 & 0,9467 & 0,8131 & 1,1022 \\
\hline
\end{tabular}

CI: confidence interval.

and distant metastases) did not exert a significant effect on ZEB2 expression (Table 3).

SLUG and ZEB2 expressions were identified as significant predictors of higher FIGO stages (III or IV) on univariate analysis $(p<0.05)$. Patients with FIGO stage III or IV were more likely to present with SLUG and ZEB2 overexpression. Multivariate analysis identified SLUG overexpres- sion as the only significant independent predictor of the higher clinical stage $(p<0.05)$ (Tables 4 and 5 ).

The overexpression of SLUG was also a significant predictor of more aggressive type II EC on both univariate and multivariate analyses $(p<0.01)$. Another independent predictor identified on the multivariate analysis was ZEB2 overexpression which turned out to be associated with a lesser likelihood of type II EC ( $p<0.05)$ (Tables 6 and 7$)$.

Univariate analysis demonstrated that the overexpression of SLUG was associated with a significantly higher likelihood of myometrial invasion $\geq 50 \%$ of the uterine wall thickness $(p<0.05)$. Multivariate analysis confirmed that the overexpression of SLUG was a significant independent predictor of the deep myometrial invasion $(p<0.01)$; the lesser likelihood of the deep myometrial invasion was in turn independently predicted by TWIST2 overexpression $(p<0.05)$ (Tables 8 and 9). The overexpression of SLUG and ZEB2 was shown to be significant predictors of adnexal involvement on univariate analysis $(p<0.05)$ (Tables 10 and 11$)$. The overexpression of SLUG was also identified as the only significant predictor of distant metastases on both univariate and multivariate analyses $(p<0.05)$ (Tables 12 and 13). None of the analyzed proteins turned out to be a significant $(p>0.05)$ predictor of LVSI, cervical invasion, and lymph node involvement.

Both univariate and multivariate analyses demonstrated that SLUG expression was the only predictor of 5-year survival in the study group. The overexpression of SLUG was 
TABLE 5: Results of multivariate logistic regression analyses examining the effects of selected proteins on the incidence of advanced FIGO stage (FIGO III+IV).

\begin{tabular}{lccccc}
\hline & $b$ coefficient & $p$ value & Odds ratio & $-95 \%$ CI & $+95 \%$ CI \\
\hline Intercept & $-2,0322$ & 0,0111 & 0,131 & 0,0273 & 0,6285 \\
TWIST 2 & 0,0412 & 0,6722 & 1,042 & 0,8611 & 1,2611 \\
TWIST 1 & $-0,2112$ & 0,3781 & 0,8096 & 0,5062 & 1,2949 \\
SLUG & 0,1036 & 0,0377 & 1,1092 & 1,0059 & 1,223 \\
ZEB 2 & 0,1375 & 0,1548 & 1,1474 & 0,9494 & 1,3866 \\
ZEB 1 & $-0,0462$ & 0,5792 & 0,9549 & 0,8111 & 1,1241 \\
SNAIL & $-0,0752$ & 0,4056 & 0,9275 & 0,7768 & 1,1075 \\
\hline
\end{tabular}

CI: confidence interval.

TABLE 6: Results of univariate logistic regression analyses examining the effects of selected proteins on the incidence of subtype 2 by Bokhman.

\begin{tabular}{lccccc}
\hline & $b$ coefficient & $p$ value & Odds ratio & $-95 \%$ CI & $+95 \%$ CI \\
\hline Intercept & $-0,657$ & 0,267 & 0,5184 & 0,1625 & 1,6539 \\
TWIST 2 & $-0,063$ & 0,4825 & 0,9389 & 0,7875 & 1,1195 \\
Intercept & $-1,0878$ & $<0,0001$ & 0,337 & 0,2313 & 0,491 \\
TWIST 1 & 0,0843 & 0,5438 & 1,0879 & 0,8287 & 1,4282 \\
Intercept & $-2,5356$ & $<0,0001$ & 0,0792 & 0,029 & 0,2161 \\
SLUG & 0,1866 & 0,0007 & 1,2052 & 1,0818 & 1,3427 \\
Intercept & $-0,4486$ & 0,3671 & 0,6385 & 0,2409 & 1,6926 \\
ZEB 2 & $-0,1212$ & 0,1991 & 0,8858 & 0,7362 & 1,0659 \\
Intercept & $-0,8881$ & 0,0193 & 0,4115 & 0,1955 & 0,8659 \\
ZEB 1 & $-0,0388$ & 0,6175 & 0,962 & 0,8262 & 1,12 \\
Intercept & $-1,2654$ & 0,0004 & 0,2821 & 0,141 & 0,5647 \\
SNAIL & 0,0579 & 0,4627 & 1,0596 & 0,9079 & 1,2366 \\
\hline
\end{tabular}

CI: confidence interval.

TABLE 7: Results of multivariate logistic regression analyses examining the effects of selected proteins on the incidence of subtype 2 by Bokhman.

\begin{tabular}{lccccc}
\hline & $b$ coefficient & $p$ value & Odds ratio & $-95 \%$ CI & $+95 \%$ CI \\
\hline Intercept & $-1,3111$ & 0,1273 & 0,2695 & 0,05 & 1,4538 \\
TWIST 2 & $-0,0371$ & 0,7225 & 0,9635 & 0,785 & 1,1827 \\
TWIST 1 & 0,1521 & 0,3927 & 1,1643 & 0,8215 & 1,6502 \\
SLUG & 0,2277 & 0,0001 & 1,2557 & 1,1192 & 1,4089 \\
ZEB 2 & $-0,2412$ & 0,0307 & 0,7857 & 0,6313 & 0,9778 \\
ZEB 1 & $-0,1183$ & 0,1987 & 0,8884 & 0,7417 & 1,0641 \\
SNAIL & 0,0999 & 0,2631 & 1,1051 & 0,9277 & 1,3165 \\
\hline
\end{tabular}

CI: confidence interval.

associated with a significant increase in mortality hazard on univariate analysis $(p<0.05)$ and was shown to be a highly significant predictor of death on multivariate analysis $(p<0.01)$ (Tables 14 and 15$)$. The probability of survival in patients with SLUG expressions $<6$ and $\geq 6$ is depicted in Figure 7. Highly significant differences in the survivals of the two groups were confirmed with log-rank, WilcoxonBreslow-Gehan, and Taron-Ware tests $(p<0.01)$.
TABLE 8: Results of univariate logistic regression analyses examining the effects of selected proteins on the incidence of myometrial invasion $>=50 \%$.

\begin{tabular}{lccccc}
\hline & $b$ coefficient & $p$ value & Odds ratio & $-95 \% \mathrm{CI}$ & $+95 \% \mathrm{CI}$ \\
\hline Intercept & 1,7835 & 0,0048 & 5,9505 & 1,7245 & 20,5328 \\
TWIST 2 & $-0,1413$ & 0,1219 & 0,8682 & 0,7258 & 1,0385 \\
Intercept & 0,877 & $<0,0001$ & 2,4036 & 1,6783 & 3,4424 \\
TWIST 1 & $-0,0397$ & 0,7758 & 0,9611 & 0,7315 & 1,2628 \\
Intercept & 0,0345 & 0,9225 & 1,0352 & 0,5161 & 2,0761 \\
SLUG & 0,1178 & 0,0108 & 1,1251 & 1,0276 & 1,2318 \\
Intercept & 1,0222 & 0,0349 & 2,7794 & 1,0753 & 7,184 \\
ZEB 2 & $-0,0309$ & 0,7225 & 0,9695 & 0,8173 & 1,1501 \\
Intercept & 0,3432 & 0,3423 & 1,4095 & 0,6941 & 2,8622 \\
ZEB 1 & 0,1223 & 0,1104 & 1,1301 & 0,9725 & 1,3132 \\
Intercept & 0,9194 & 0,006 & 2,5079 & 1,3015 & 4,8324 \\
SNAIL & $-0,0178$ & 0,8159 & 0,9824 & 0,846 & 1,1408 \\
\hline
\end{tabular}

CI: confidence interval.

TABLE 9: Results of multivariate logistic regression analyses examining the effects of selected proteins on the incidence of myometrial invasion $>=50 \%$.

\begin{tabular}{lccccc}
\hline & $b$ coefficient & $p$ value & Odds ratio & $-95 \%$ CI & $+95 \%$ CI \\
\hline Intercept & 0,8942 & 0,2557 & 2,4455 & 0,5232 & 11,4298 \\
TWIST 2 & $-0,2234$ & 0,0345 & 0,7998 & 0,6502 & 0,9838 \\
TWIST 1 & $-0,123$ & 0,4994 & 0,8843 & 0,6189 & 1,2635 \\
SLUG & 0,133 & 0,0085 & 1,1423 & 1,0345 & 1,2614 \\
ZEB 2 & $-0,0185$ & 0,8488 & 0,9817 & 0,8117 & 1,1873 \\
ZEB 1 & 0,1679 & 0,0548 & 1,1829 & 0,9966 & 1,4039 \\
SNAIL & $-0,0276$ & 0,7500 & 0,9728 & 0,8211 & 1,1526 \\
\hline
\end{tabular}

CI: confidence interval.

\section{Discussion}

Tumor microenvironment parameters, in particular, changes in tumor cells-stromal cells interactions, play a crucial role in the development of epithelial malignancies [34]. Mesenchymal cells are essential for the control of epithelial growth, differentiation and function, and abnormal mesenchymalepithelial interactions found in various human tumors, including EC [35, 36]. Metastatic spread results from a series of linked, sequential, and selective steps involving cell migration, invasion, adhesion, and proliferation, as well as angiogenesis. Invasion, which is a critical step in the metastatic cascade, requires the interaction of tumor cells with their environment [37]. EMT is the essential initial step of malignant transformation, during which the cells acquire invasiveness and metastatic potential by losing epithelial polarity and reducing intercellular adhesion [38, 39]. Many studies showed that EMT is involved in tumorigenesis and development of EC [40]. Downregulation of E-cadherin is considered a classical molecular switch for EMT and has been implicated in the invasion and spread of EC [41, 42].

Several transcription factors, among them the members of SLUG, SNAIL, ZEB, and TWIST families, have been 
TABLE 10: Results of univariate logistic regression analyses examining the effects of selected proteins on the incidence of infiltration of adnexa.

\begin{tabular}{lccccc}
\hline & $b$ coefficient & $p$ value & Odds ratio & $-95 \%$ CI & $+95 \%$ CI \\
\hline Intercept & $-2,1811$ & 0,0098 & 0,1129 & 0,0216 & 0,5912 \\
TWIST 2 & 0,0463 & 0,7067 & 1,0474 & 0,8229 & 1,3333 \\
Intercept & $-1,8675$ & $<0,0001$ & 0,1545 & 0,0949 & 0,2515 \\
TWIST 1 & $-0,0368$ & 0,862 & 0,9639 & 0,6365 & 1,4597 \\
Intercept & $-3,0314$ & $<0,0001$ & 0,0482 & 0,0131 & 0,1771 \\
SLUG & 0,1438 & 0,0408 & 1,1546 & 1,006 & 1,3252 \\
Intercept & $-3,2094$ & $<0,0001$ & 0,0404 & 0,0094 & 0,1733 \\
ZEB 2 & 0,2418 & 0,0437 & 1,2736 & 1,0068 & 1,6109 \\
Intercept & $-1,3751$ & 0,0033 & 0,2528 & 0,1011 & 0,632 \\
ZEB 1 & $-0,1218$ & 0,2366 & 0,8853 & 0,7237 & 1,0831 \\
Intercept & $-1,9968$ & $<0,0001$ & 0,1358 & 0,0527 & 0,3496 \\
SNAIL & 0,0332 & 0,7617 & 1,0337 & 0,8343 & 1,2807 \\
\hline
\end{tabular}

CI: confidence interval.

TABLE 11: Results of multivariate logistic regression analyses examining the effects of selected proteins on the incidence of infiltration of adnexa.

\begin{tabular}{lccccc}
\hline & $b$ coefficient & $p$ value & Odds ratio & $-95 \%$ CI & $+95 \%$ CI \\
\hline Intercept & $-3,4897$ & 0,0026 & 0,0305 & 0,0031 & 0,2961 \\
TWIST 2 & $-0,0102$ & 0,9412 & 0,9899 & 0,7554 & 1,2972 \\
TWIST 1 & 0,028 & 0,9102 & 1,0284 & 0,6319 & 1,6738 \\
SLUG & 0,1343 & 0,0619 & 1,1438 & 0,9934 & 1,317 \\
ZEB 2 & 0,1805 & 0,1718 & 1,1978 & 0,9246 & 1,5517 \\
ZEB 1 & $-0,1547$ & 0,2146 & 0,8567 & 0,671 & 1,0937 \\
SNAIL & 0,0667 & 0,6059 & 1,069 & 0,8298 & 1,3771 \\
\hline
\end{tabular}

CI: confidence interval.

TABLE 12: Results of univariate logistic regression analyses examining the effects of selected proteins on the incidence of distant metastases.

\begin{tabular}{lccccc}
\hline & $b$ coefficient & $p$ value & Odds ratio & $-95 \%$ CI & $+95 \%$ CI \\
\hline Intercept & $-2,8899$ & 0,0045 & 0,0556 & 0,0076 & 0,4076 \\
TWIST 2 & 0,0967 & 0,5085 & 1,1015 & 0,8271 & 1,4669 \\
Intercept & $-2,1927$ & $<0,0001$ & 0,1116 & 0,0634 & 0,1964 \\
TWIST 1 & $-0,277$ & 0,5042 & 0,7581 & 0,3363 & 1,7088 \\
Intercept & $-4,1797$ & $<0,0001$ & 0,0153 & 0,0024 & 0,0972 \\
SLUG & 0,2256 & 0,0153 & 1,253 & 1,0441 & 1,5037 \\
Intercept & $-2,767$ & 0,0006 & 0,0628 & 0,0129 & 0,3063 \\
ZEB 2 & 0,0958 & 0,4898 & 1,1006 & 0,8385 & 1,4444 \\
Intercept & $-2,926$ & $<0,0001$ & 0,0536 & 0,0148 & 0,1944 \\
ZEB 1 & 0,1408 & 0,2304 & 1,1512 & 0,9146 & 1,449 \\
Intercept & $-2,2616$ & $<0,0001$ & 0,1042 & 0,0369 & 0,2939 \\
SNAIL & 0,0028 & 0,9816 & 1,0028 & 0,7915 & 1,2705 \\
\hline
\end{tabular}

CI: confidence interval.

implicated in the transcriptional repression of E-cadherin in a broad spectrum of human cancers and were shown to be associated with aggressive tumor behavior and poor progno-
TABLE 13: Results of multivariate logistic regression analyses examining the effects of selected proteins on the incidence of distant metastases.

\begin{tabular}{lccccc}
\hline & $b$ coefficient & $p$ value & Odds ratio & $-95 \%$ CI & $+95 \%$ CI \\
\hline Intercept & $-5,1986$ & 0,0012 & 0,0055 & 0,0002 & 0,1295 \\
TWIST 2 & 0,0514 & 0,7505 & 1,0528 & 0,7668 & 1,4455 \\
TWIST 1 & $-0,3348$ & 0,4795 & 0,7155 & 0,2828 & 1,8098 \\
SLUG & 0,2357 & 0,0171 & 1,2658 & 1,0428 & 1,5363 \\
ZEB 2 & 0,0326 & 0,8438 & 1,0332 & 0,7467 & 1,4295 \\
ZEB 1 & 0,1489 & 0,2665 & 1,1606 & 0,8925 & 1,5093 \\
SNAIL & $-0,056$ & 0,6888 & 0,9455 & 0,7187 & 1,2438 \\
\hline
\end{tabular}

CI: confidence interval.

TABle 14: Prognostic factors for overall survival selected by Cox's univariate analysis.

\begin{tabular}{lccccc}
\hline & $b$ coefficient & $p$ value & HR & $-95 \%$ CI & $+95 \%$ CI \\
\hline TWIST 2 & 0,0623 & 0,4678 & 1,0643 & 0,8995 & 1,2592 \\
TWIST 1 & $-14,149$ & 0,9875 & 0,0000 & 0,0000 & 0,0000 \\
SLUG & 0,1157 & 0,0138 & 1,1226 & 1,0239 & 1,2309 \\
ZEB 2 & $-0,0274$ & 0,7393 & 0,973 & 0,828 & 1,1434 \\
ZEB 1 & 0,0558 & 0,4176 & 1,0574 & 0,9239 & 1,2102 \\
SNAIL & 0,0363 & 0,6143 & 1,037 & 0,9004 & 1,1943 \\
\hline
\end{tabular}

CI: confidence interval; HR: hazard ratio.

Table 15: Prognostic factors for overall survival selected by Cox's multivariate analysis.

\begin{tabular}{lccccc}
\hline & $b$ coefficient & $p$ value & HR & $-95 \%$ CI & $+95 \%$ CI \\
\hline TWIST 2 & 0,0479 & 0,601 & 1,049 & 0,8767 & 1,2552 \\
TWIST 1 & $-13,63$ & 0,9816 & $1,2 \mathrm{E}-6$ & 0 & NA \\
SLUG & 0,1374 & 0,0052 & 1,1472 & 1,0418 & 1,2633 \\
ZEB 2 & $-0,1108$ & 0,2217 & 0,8951 & 0,7494 & 1,0692 \\
ZEB 1 & 0,0126 & 0,862 & 1,0127 & 0,8783 & 1,1677 \\
SNAIL & 0,0648 & 0,4437 & 1,0669 & 0,904 & 1,2592 \\
\hline
\end{tabular}

CI: confidence interval; HR: hazard ratio.

sis [43-45]. In our present study, the expression of the EMT transcription factors was found in the vast majority of tissue samples, which implies that EMT plays a critical role in endometrial carcinogenesis. Moreover, the expression of SLUG turned out to be significantly higher in patients with FIGO stages III and IV, as well as in those with type II EC. It needs to be emphasized that we determined the expressions of EMT transcription factors in a relatively large group of patients with advanced EC. EMT, promoted by several transcription factors, causes downregulation of epithelial marker genes and contributes to the establishment of a mesenchymal phenotype [46]. SNAIL and SLUG, belonging to the group of zinc finger-type proteins, are regarded as major EMT inducers that inhibit the transcription of cell adhesion molecules, among them is E-cadherin [47]. As potent Ecadherin repressors, those proteins contribute to the loss of tight junctions between epithelial cells and initiate EMT, which facilitates cancer cell invasion and formation of distant 


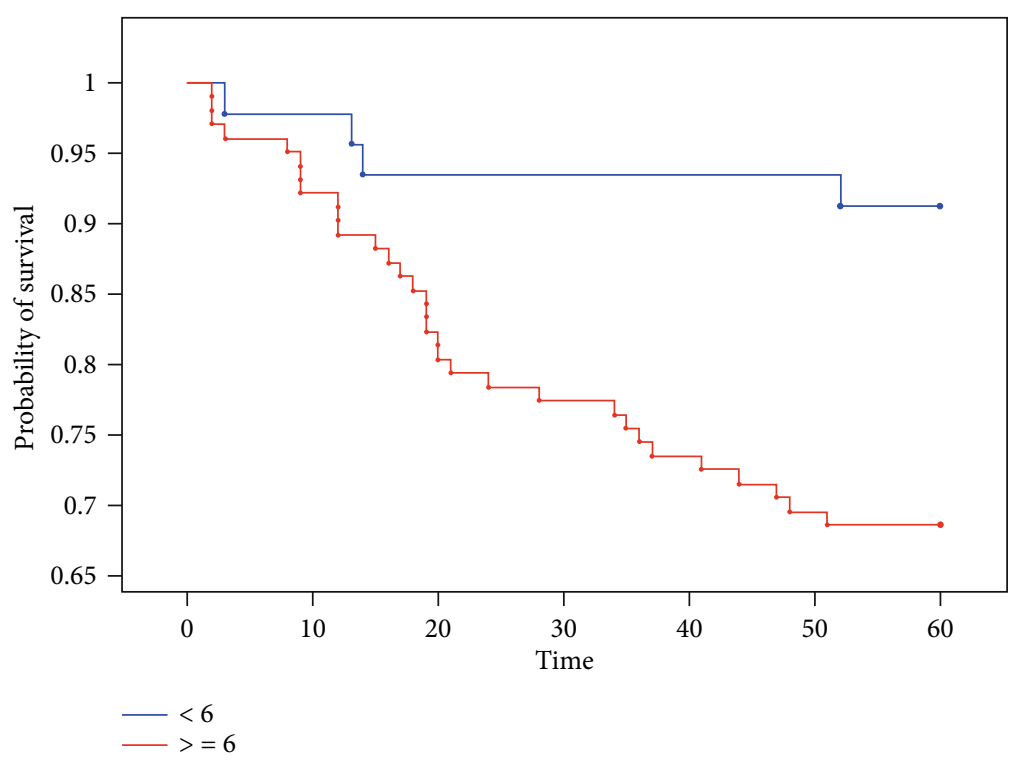

Figure 7: Kaplan-Meier curves for overall survival in patients with endometrial cancer stratified by SLUG expression. The Kaplan-Meier plots illustrate statistically significant differences $(p<0.05)$ in the probability of survival in patients with SLUG expression $\geq 6$ and $<6$ (time/months). Logrank $\mathrm{chi}^{2}=8,2801, \mathrm{df}=1$, and $p=0.0040$. Wilcoxon-Breslow-Gehanchi ${ }^{2}=8,0659, \mathrm{df}=1, \quad$ and $p=0.0045$. TaronWarechi $^{2}=8,1876, \mathrm{df}=1$, and $p=0,0042$.

metastases $[48,49]$. The EMT signaling pathways may be activated by several cytokines or growth factors present in the local microenvironment, followed by the interaction with transforming growth factor-beta (TGF- $\beta$ ), bone morphogenetic protein, Wnt/ $\beta$-catenin pathway, Notch, Hedgehog, and RTKs $[47,50]$. Some signaling pathways, such as the phosphoinositide 3-kinase/protein kinase B (PI3K/AKT) pathway, upregulate SNAIL and SLUG, whereas the estrogen receptor is known to repress the transcription of those proteins $[51,52]$. Importantly, the overexpression of SLUG can be mediated by various mechanisms; for example, $17 \mathrm{~b}$ estradiol is known to increase the expression of vimentin and SLUG and to decrease the expression of E-cadherin, which leads to the inhibition of EMT and estrogen-induced EMT in EC cells [53]. Also, transforming growth factorbeta (TGF- $\beta$ ) was shown to be an EMT inducer which downregulates E-cadherin, upregulates SLUG, and stimulates cell invasion in EC; the TGF-b-induced cell invasion could be prevented through SLUG depletion caused by siRNA knockdown [54].

Our present study demonstrated that myometrial invasion exceeding $50 \%$ of the uterine wall thickness and adnexal involvement were associated with a significant increase in SLUG expression. In turn, the overexpression of TWIST2 correlated with a lesser risk of deep myometrial infiltration in EC patients. A significant increase in SLUG expression was also observed in patients with distant metastases. Adnexal involvement was also shown to be associated with a significant increase in ZEB2 expression. Our findings are consistent with the results published by other authors, according to whom the deep myometrial invasion was associated with SLUG overexpression and a concomitant decrease in E-cadherin level [55]. Recent studies showed that SLUG plays a role not only in cancer spread but also in cancer stemness [56, 57]; this sug- gests that the protein might be involved in the early stages of cancer progression. SLUG is known to suppress both p53dependent and p53-independent apoptotic pathways [58]. Tumors expressing SLUG might display some characteristics of cancer stem cells, such as therapeutic resistance and ability to recur. The overexpression of E-cadherin transcription repressors, TWIST, SNAIL, and SLUG, was observed in both EC cell lines and tumor samples, and downregulation of Ecadherin was demonstrated in either endometrioid or nonendometrioid ECs. Hence, SLUG might play a significant role in the development of therapeutic resistance and contribute to poor prognosis in a subset of high-grade ECs. Despite complete resection, patients with the extrauterine disease are assumed to present with micrometastases, and thus, are at increased risk of tumor recurrence; if the primary malignancy overexpresses SLUG, the recurrent tumor is likely to be resistant to adjuvant therapy [59]. ZEB1 and ZEB2 are transcriptional repressors of E-cadherin in a miR-200-dependent mechanism. The inhibition of miR-200 results in the downregulation of E-cadherin through upregulation of its transcriptional repressors, ZEB1 and ZEB2. Hence, the ZEB proteins and miR-200 are considered a driving force for cancer progression and spread by controlling the state of cancer stem cells $[60,61]$.

In our present study, SLUG was the only protein, the expression of which was identified as a significant predictor of 5-year survival on both univariate and multivariate analyses. The overexpression of SLUG was associated with a significant increase in mortality hazard. This implies that the expression of SLUG may be an important determinant of survival in EC. Some clinicopathological parameters, identified as independent predictors of overall survival and disease-free survival in EC, among them patient age, tumor grade, histological type, and LVSI, are considered decisive 
factors during the selection of surgical treatment and adjuvant therapy options [62]. Established prognostic factors for the recurrence and spread of EC include surgical FIGO stage, tumor grade, histological type, and myometrial and lymphovascular invasion $[63,64]$. Our findings suggest that the inclusion of SLUG and ZEB2 expressions in the armamentarium of routinely performed immunohistochemical tests might contribute to more accurate prognosis and facilitate the planning of adjuvant therapy, especially in patients with advanced clinical stages of type I EC and those with type II malignancies.

\section{Conclusions}

(1) Selected proteins of the EMT pathway play a role in endometrial carcinogenesis

(2) SLUG and ZEB2 expressions in the primary tumor might predict clinical outcomes in EC and drive therapeutic decisions regarding adjuvant treatment in patients with this malignancy

\section{Data Availability}

The data used to support the findings of this study are available from the corresponding author upon request.

\section{Conflicts of Interest}

None is declared.

\section{Acknowledgments}

The authors would like to acknowledge Prof. Marek Grabiec and Dr. Hab Dariusz Grzanka for their keen interest, inspiring guidance, and consist encouragement.

\section{References}

[1] J. Ferlay, I. Soerjomataram, R. Dikshit et al., "Cancer incidence and mortality worldwide: sources, methods and major patterns in GLOBOCAN 2012," International Journal of Cancer, vol. 136, no. 5, pp. E359-E386, 2015.

[2] R. L. Siegel, K. D. Miller, and A. Jemal, "Cancer statistics, 2017," CA: a Cancer Journal for Clinicians, vol. 67, no. 1, pp. 7-30, 2017.

[3] J. Lortet-Tieulent, J. Ferlay, F. Bray, and A. Jemal, "International patterns and trends in endometrial cancer incidence, 1978-2013," Journal of the National Cancer Institute, vol. 110, no. 4, pp. 354-361, 2018.

[4] C. R. Smittenaar, K. A. Petersen, K. Stewart, and N. Moitt, "Cancer incidence and mortality projections in the UK until 2035," British Journal of Cancer, vol. 115, no. 9, pp. 11471155, 2016.

[5] M. A. Sheikh, A. D. Althouse, K. E. Freese et al., "USA endometrial cancer projections to 2030: should we be concerned?" Future Oncology, vol. 10, no. 16, pp. 2561-2568, 2014.

[6] R. L. Siegel, S. S. Devesa, V. Cokkinides, J. Ma, and A. Jemal, "State-level uterine corpus cancer incidence rates corrected for hysterectomy prevalence, 2004 to 2008," Cancer Epidemiology, Biomarkers \& Prevention, vol. 22, no. 1, pp. 25-31, 2013.
[7] C. E. DeSantis, C. C. Lin, A. B. Mariotto et al., "Cancer treatment and survivorship statistics, 2014," CA: A Cancer Journal for Clinicians, vol. 64, no. 4, pp. 252-271, 2014.

[8] A. Yeramian, G. Moreno-Bueno, X. Dolcet et al., "Endometrial carcinoma: molecular alterations involved in tumor development and progression," Oncogene, vol. 32, no. 4, pp. 403413, 2013.

[9] F. Bray, J. Ferlay, I. Soerjomataram, R. L. Siegel, L. A. Torre, and A. Jemal, "Global cancer statistics 2018: GLOBOCAN estimates of incidence and mortality worldwide for 36 cancers in 185 countries," CA: A Cancer Journal for Clinicians, vol. 68, no. 6, pp. 394-424, 2018.

[10] E. Weiderpass, J. Antoine, F. I. Bray, J. K. Oh, and M. Arbyn, "Trends in corpus uteri cancer mortality in member states of the European Union," European Journal of Cancer, vol. 50, no. 9, pp. 1675-1684, 2014.

[11] L. A. Brinton, A. S. Felix, D. S. McMeekin et al., "Etiologic heterogeneity in endometrial cancer: evidence from a Gynecologic Oncology Group trial," Gynecologic Oncology, vol. 129, no. 2, pp. 277-284, 2013.

[12] J. V. Bokhman, "Two pathogenetic types of endometrial carcinoma," Gynecologic Oncology, vol. 15, no. 1, pp. 10-17, 1983.

[13] A. Alkushi, Z. H. Abdul-Rahman, P. Lim et al., "Description of a novel system for grading of endometrial carcinoma and comparison with existing grading systems," The American Journal of Surgical Pathology, vol. 29, no. 3, pp. 295-304, 2005.

[14] A. N. Scholten, V. T. Smit, H. Beerman, W. L. van Putten, and C. L. Creutzberg, "Prognostic significance and interobserver variability of histologic grading systems for endometrial carcinoma," Cancer, vol. 100, no. 4, pp. 764-772, 2004.

[15] P. T. Soliman, J. C. Oh, K. M. Schmeler et al., "Risk factors for young premenopausal women with endometrial cancer," Obstetrics and Gynecology, vol. 105, no. 3, pp. 575-580, 2005.

[16] V. W. Setiawan, H. P. Yang, M. C. Pike et al., "Type I and II endometrial cancers: have they different risk factors?," Journal of Clinical Oncology, vol. 31, no. 20, pp. 2607-2618, 2013.

[17] M. P. Madigan, R. Troisi, N. Potischman, J. F. Dorgan, L. A. Brinton, and R. N. Hoover, "Serum hormone levels in relation to reproductive and lifestyle factors in postmenopausal women (United States)," Cancer Causes \& Control, vol. 9, no. 2, pp. 199-207, 1998.

[18] S. Topuz, H. Sozen, D. Vatansever et al., "Do obesity and age effect the clinicopathological features and survival outcomes in premenopausal women with endometrial cancer?," European Journal of Gynaecological Oncology, vol. 37, no. 3, pp. 320-326, 2016.

[19] S. J. Schonfeld, P. Hartge, R. M. Pfeiffer et al., "An aggregated analysis of hormonal factors and endometrial cancer risk by parity," Cancer, vol. 119, no. 7, pp. 1393-1401, 2013.

[20] H. P. Yang, N. Wentzensen, B. Trabert et al., "Endometrial cancer risk factors by 2 main histologic subtypes," American Journal of Epidemiology, vol. 177, no. 2, pp. 142-151, 2013.

[21] V. W. Setiawan, M. C. Pike, S. Karageorgi et al., "Age at last birth in relation to risk of endometrial cancer: pooled analysis in the epidemiology of endometrial cancer consortium," American Journal of Epidemiology, vol. 176, no. 4, pp. 269278, 2012.

[22] Uterine neoplasms, NCCN Clinical Practice Guidelines in OncologyDecember 2016. https://www.nccn.org/professionals/ physician_gls/pdf/uterine.pdf. 
[23] J. D. Wright, N. I. B. Medel, J. Sehouli, K. Fujiwara, and T. J. Herzog, "Contemporary management of endometrial cancer," Lancet, vol. 379, no. 9823, pp. 1352-1360, 2012.

[24] A. Mariani, S. C. Dowdy, W. A. Cliby et al., "Prospective assessment of lymphatic dissemination in endometrial cancer: a paradigm shift in surgical staging," Gynecologic Oncology, vol. 109, no. 1, pp. 11-18, 2008.

[25] H. Gao and Z. Zhang, "Laparoscopy versus laparotomy in the treatment of high-risk endometrial cancer," Medicine, vol. 94, no. 30, article e1245, 2015.

[26] A. Mariani, M. J. Webb, L. Galli, and K. C. Podratz, "Potential therapeutic role of para-aortic lymphadenectomy in nodepositive endometrial cancer," Gynecologic Oncology, vol. 76, no. 3, pp. 348-356, 2000.

[27] G. H. Eltabbakh, M. S. Piver, R. E. Hempling, and K. H. Shin, "Excellent long-term survival and absence of vaginal recurrences in 332 patients with low-risk stage I endometrial adenocarcinoma treated with hysterectomy and vaginal brachytherapy without formal staging lymph node sampling: report of a prospective trial," International Journal of Radiation Oncology • Biology • Physics, vol. 38, no. 2, pp. 373-380, 1997.

[28] A. Mariani, M. J. Webb, G. L. Keeney, M. G. Haddock, G. Calori, and K. C. Podratz, "Low-risk corpus cancer: is lymphadenectomy or radiotherapy necessary?" American Journal of Obstetrics and Gynecology, vol. 182, no. 6, pp. 1506-1519, 2000.

[29] L. Larue and A. Bellacosa, "Epithelial-mesenchymal transition in development and cancer: role of phosphatidylinositol $3^{\prime}$ kinase/AKT pathways," Oncogene, vol. 24, no. 50, pp. $7443-$ $7454,2005$.

[30] E. Colas, N. Pedrola, L. Devis et al., "The EMT signaling pathways in endometrial carcinoma," Clinical and Translational Oncology, vol. 14, no. 10, pp. 715-720, 2012.

[31] J. J. Sznurkowski, P. Knapp, L. Bodnar et al., "Recommendations of the Polish Gynecological Oncology Society for the diagnosis andtreatment of endometrial cancer," Current Gynecologic Oncology, vol. 15, no. 1, pp. 34-44, 2017.

[32] P. Sadlecki, J. Jóźwicki, P. Antosik, and M. Grabiec, "Expression of selected epithelial-mesenchymal transition transcription factors in serous borderline ovarian tumors and type I ovarian cancers," Tumor Biology, vol. 40, no. 6, article 101042831878480, 2018.

[33] W. Remmele and H. E. Stegner, "Recommendation for uniform definition of an immunoreactive score (IRS) for immunohistochemical estrogen receptor detection (ER-ICA) in breast cancer tissue," Pathologe, vol. 8, no. 3, pp. 138-140, 1987.

[34] M. Shi, H. Zhang, M. Li et al., "Normal endometrial stromal cells regulate survival and apoptosis signaling through PI3K/AKt/ Survivin pathway in endometrial adenocarcinoma cells in vitro," Gynecologic Oncology, vol. 123, no. 2, pp. 387392, 2011.

[35] P. Sadlecki, M. Grabiec, D. Grzanka, J. Jóźwicki, P. Antosik, and M. Walentowicz-Sadlecka, "Expression of zinc finger transcription factors (ZNF143 and ZNF281) in serous borderline ovarian tumors and low-grade ovarian cancers," J Ovarian Res, vol. 12, no. 1, p. 23, 2019.

[36] T. Arnold Julia, A. Lessey Bruce, M. Seppala, and D. G. Kaufman, "Effect of normal endometrial stroma on growth and differentiation in Ishikawa endometrial adenocarcinoma cells," Cancer Research, vol. 62, pp. 79-88, 2002.
[37] E. C. Woodhouse, R. F. Chuaqui, and L. A. Liotta, "General mechanisms of metastasis," Cancer, vol. 80, no. S8, pp. 1529S1537, 1997.

[38] M. Tania, M. A. Khan, and J. Fu, "Epithelial to mesenchymal transition inducing transcription factors and metastatic cancer," Tumor Biology, vol. 35, no. 8, pp. 7335-7342, 2014.

[39] J. J. Christiansen and A. K. Rajasekaran, "Reassessing epithelial to mesenchymal transition as a prerequisite for carcinoma invasion and metastasis," Cancer Research, vol. 66, no. 17, pp. 8319-8326, 2006.

[40] M. Singh, N. S. Spoelstra, A. Jean et al., "ZEB1 expression in type I vs type II endometrial cancers: a marker of aggressive disease," Modern Pathology, vol. 21, no. 7, pp. 912-923, 2008 .

[41] S. Matsuzaki and C. Darcha, "Epithelial to mesenchymal transition-like and mesenchymal to epithelial transition-like processes might be involved in the pathogenesis of pelvic endometriosis," Human Reproduction, vol. 27, no. 3, pp. 712-721, 2012.

[42] A. Cano, M. A. Pérez-Moreno, I. Rodrigo et al., "The transcription factor Snail controls epithelial-mesenchymal transitions by repressing E-cadherin expression," Nature Cell Biology, vol. 2, no. 2, pp. 76-83, 2000.

[43] H. Peinado, D. Olmeda, and A. Cano, "Snail, Zeb and bHLH factors in tumour progression: an alliance against the epithelial phenotype?" Nature Reviews. Cancer, vol. 7, no. 6, pp. 415$428,2007$.

[44] A. G. de Herreros, S. Peiró, M. Nassour, and P. Savagner, "Snail family regulation and epithelial mesenchymal transitions in breast cancer progression," Journal of Mammary Gland Biology and Neoplasia, vol. 15, no. 2, pp. 135-147, 2010.

[45] E. Sánchez-Tilló, Y. Liu, O. de Barrios et al., "EMT-activating transcription factors in cancer: beyond EMT and tumor invasiveness," Cellular and Molecular Life Sciences, vol. 69, no. 20, pp. 3429-3456, 2012.

[46] Y. Tanaka, Y. Terai, H. Kawaguchi et al., "Prognostic impact of EMT (epithelial-mesenchymal-transition)-related protein expression in endometrial cancer," Cancer Biology \& Therapy, vol. 14, no. 1, pp. 13-19, 2014.

[47] D. M. Gonzalez and D. Medici, "Signaling mechanisms of the epithelial-mesenchymal transition," Sci Signal, vol. 7, no. 344, p. re8, 2014.

[48] V. Bolos, H. Peinado, M. A. Perez-Moreno, M. F. Fraga, M. Esteller, and A. Cano, "The transcription factor Slug represses E-cadherin expression and induces epithelial to mesenchymal transitions: a comparison with Snail and E47 repressors," Journal of Cell Science, vol. 116, no. 3, pp. 499-511, 2002.

[49] P. B. Gupta, C. Kuperwasser, J. P. Brunet et al., "The melanocyte differentiation program predisposes to metastasis after neoplastic transformation," Nature Genetics, vol. 37, no. 10, pp. 1047-1054, 2005.

[50] L. Campo, C. Zhang, and E. K. Breuer, "EMT-Inducing Molecular Factors in Gynecological Cancers," BioMed Research International, vol. 2015, Article 420891, pp. 1-12, 2015.

[51] A. Dhasarathy, M. Kajita, and P. A. Wade, "The transcription factor snail mediates epithelial to mesenchymal transitions by repression of estrogen receptor-alpha," Molecular Endocrinology, vol. 21, no. 12, pp. 2907-2918, 2007.

[52] Y. Ye, Y. Xiao, W. Wang et al., "ERalpha signaling through slug regulates E-cadherin and EMT," Oncogene, vol. 29, no. 10 , pp. 1451-1462, 2010. 
[53] H. Zhang, H. Li, S. Qi et al., "Normal endometrial stromal cells regulate $17 \beta$-estradiol-induced epithelial-mesenchymal transition via SLUG and E-cadherin in endometrial adenocarcinoma cells in vitro," Gynecological Endocrinology, vol. 33, no. 1, pp. 82-86, 2016.

[54] M. Huszar, M. Pfeifer, U. Schirmer et al., "Up-regulation of L1CAM is linked to loss of hormone receptors and Ecadherin in aggressive subtypes of endometrial carcinomas," The Journal of Pathology, vol. 220, no. 5, pp. 551-561, 2010.

[55] N. Montserrat, A. Mozos, D. Llobet et al., "Epithelial to mesenchymal transition in early stage endometrioid endometrial carcinoma," Human Pathology, vol. 43, no. 5, pp. 632-643, 2012.

[56] G. Storci, P. Sansone, D. Trere et al., "The basal-like breast carcinoma phenotype is regulated by SLUG gene expression," The Journal of Pathology, vol. 214, no. 1, pp. 25-37, 2008.

[57] C. Yao, L. Su, J. Shan et al., "IGF/STAT3/NANOG/slug signaling axis simultaneously controls epithelial-mesenchymal transition and stemness maintenance in colorectal cancer," Stem Cells, vol. 34, no. 4, pp. 820-831, 2016.

[58] W. S. Wu, S. Heinrichs, D. Xu et al., "Slug antagonizes p53mediated apoptosis of hematopoietic progenitors by repressing puma," Cell, vol. 123, no. 4, pp. 641-653, 2005.

[59] A. Singh and J. Settleman, "EMT, cancer stem cells and drug resistance: an emerging axis of evil in the war on cancer," Oncogene, vol. 29, no. 34, pp. 4741-4751, 2010.

[60] Y. Liu, F. Nan, K. Lu et al., "Identification of key genes in endometrioid endometrial adenocarcinoma via TCGA database," Cancer Biomarkers, vol. 21, no. 1, pp. 11-21, 2017.

[61] L. Alonso-Alconada, L. Muinelo-Romay, K. Madissoo et al., "Molecular profiling of circulating tumor cells links plasticity to the metastatic process in endometrial cancer," Molecular Cancer, vol. 13, no. 1, pp. 1-10, 2014.

[62] C. L. Creutzberg, W. L. van Putten, P. C. Koper et al., "Surgery and postoperative radiotherapy versus surgery alone for patients with stage- 1 endometrial carcinoma: multicentre randomised trial," Lancet, vol. 355, no. 9213, pp. 1404-1411, 2000.

[63] C. L. Kosary, "FIGO stage, histology, histologic grade, age and race as prognostic factors in determining survival for cancers of the female gynecological system: an analysis of 1973-87 SEER cases of cancers of the endometrium, cervix, ovary, vulva, and vagina," Seminars in Surgical Oncology, vol. 10, no. 1, pp. 31-46, 1994.

[64] J. Lindauer, J. M. Fowler, T. P. Manolitsas et al., "Is there a prognostic difference between depth of myometrial invasion and the tumor-free distance from the uterine serosa in endometrial cancer?," Gynecologic Oncology, vol. 91, no. 3, pp. 547-551, 2003. 